\title{
Some Results on Strict Graded Categorical Groups
}

\author{
Nguyen Tien Quang' and Che Thi Kim Phung ${ }^{2}$ \\ ${ }^{1}$ Department of Mathematics, Hanoi National University of Education, Hanoi 123105, Vietnam \\ ${ }^{2}$ Department of Mathematics and Applications, Saigon University, Ho Chi Minh City 700900, Vietnam
}

Correspondence should be addressed to Nguyen Tien Quang; cn.nguyenquang@gmail.com

Received 21 March 2013; Accepted 15 July 2013

Academic Editor: Adolfo Ballester-Bolinches

Copyright (C) 2013 N. Tien Quang and C. Thi Kim Phung. This is an open access article distributed under the Creative Commons Attribution License, which permits unrestricted use, distribution, and reproduction in any medium, provided the original work is properly cited.

\begin{abstract}
We present some applications of strict graded categorical groups to the construction of the obstruction of an equivariant kernel and to the classification of equivariant group extensions which are central ones. The composition of a graded categorical group and an equivariant group homomorphism is also determined.
\end{abstract}

\section{Introduction}

The group extension problem has an important significance in the development of modern algebra. Some notions of this problem such as crossed product, factor set, and obstruction (see [1]) are not only applied to rings or to algebraic types but also are raised to a categorical level.

The theory of graded categorical groups studied by Cegarra et al. [2] can be viewed as a generalization of both the categorical group theory of Sinh [3] and the graded category theory of Fröhlich and Wall [4]. The equivariant group extension problem is one of applications of this theory.

Strict graded categorical groups, with their simple structures compared to the general case, are more likely to give a lot of interesting applications. In [5] we presented an application of this notion to the classification of equivariant crossed modules. In this paper we continue to introduce some other applications. Firstly, we show that if $[h]$ is the third invariant of the strict graded categorical group $\mathrm{Hol}_{\Gamma} G$ and $p: \Pi \rightarrow$ Out $G$ is an equivariant kernel; then

$$
p^{*} h \in \operatorname{Obs}(p) \text {. }
$$

Secondly, we classify equivariant group extensions of $A$ by $G$ which are central extensions by graded monoidal autofunctors of the strict graded categorical group $\int_{\Gamma}(G, A, 0)$. Finally, we construct the composition of a $\Gamma$-graded categorical group with a $\Gamma$-homomorphism, which is analogous to the composition of a group extension with a group homomorphism (see [1, Chapter 3]).

\section{Preliminaries}

2.1. Graded Categorical Groups. We recall briefly some basic notions about graded categorical groups in [2].

We regard the group $\Gamma$ as a category with one object, say *, where the morphisms are elements of $\Gamma$ and the composition is the group operation. A category $\mathbb{G}$ is $\Gamma$-graded if there is a functor $g r: \mathbb{G} \rightarrow \Gamma$. The grading $g r$ is said to be stable if for any object $X \in \mathrm{Ob} \mathbb{G}$ and any $\sigma \in \Gamma$ there exists an isomorphism $f$ in $\mathbb{G}$ with domain $X$ and $g r(f)=\sigma$. A $\Gamma$ graded monoidal category $\mathbb{G}=(\mathbb{G}, g r, \otimes, I, \mathbf{a}, \mathbf{l}, \mathbf{r})$ consists of

(1) a stable $\Gamma$-graded category $(\mathbb{G}, g r), \Gamma$-graded functors $\otimes: \mathbb{G} \times_{\Gamma} \mathbb{G} \rightarrow \mathbb{G}$ and $I: \Gamma \rightarrow \mathbb{G}$,

(2) natural isomorphisms of grade $1 \mathbf{a}_{X, Y, Z}:(X \otimes Y) \otimes$ $Z \stackrel{\sim}{\rightarrow} X \otimes(Y \otimes Z), \mathbf{l}_{X}: I \otimes X \stackrel{\sim}{\rightarrow} X$ and $\mathbf{r}_{X}: X \otimes I \stackrel{\sim}{\rightarrow} X$ such that, for all $X, Y, Z, T \in \mathrm{Ob} \mathbb{G}$, the following two coherence conditions hold:

$$
\begin{aligned}
\mathbf{a}_{X, Y, Z \otimes T} & \mathbf{a}_{X \otimes Y, Z, T} \\
= & \left(\mathrm{id}_{X} \otimes \mathbf{a}_{Y, Z, T}\right) \mathbf{a}_{X, Y \otimes Z, T}\left(\mathbf{a}_{X, Y, Z} \otimes \mathrm{id}_{T}\right), \\
& \left(\mathrm{id}_{X} \otimes \mathbf{l}_{Y}\right) \mathbf{a}_{X, I, Y}=\mathbf{r}_{X} \otimes \mathrm{id}_{Y} .
\end{aligned}
$$


A graded categorical group is a graded monoidal category $\mathbb{G}$ in which every object is invertible and every morphism is an isomorphism. In this case, the subcategory Ker $\mathbb{G}$ consisting of all objects of $\mathbb{G}$ and all morphisms of grade 1 in $\mathbb{G}$ is a categorical group.

If $\mathbb{G}, \mathbb{G}^{\prime}$ are $\Gamma$-monoidal categories, then a graded monoidal functor $\left(F, \widetilde{F}, F_{*}\right): \mathbb{G} \rightarrow \mathbb{G}^{\prime}$ consists of a $\Gamma$-graded functor $F: \mathbb{G} \rightarrow \mathbb{G}^{\prime}$, natural isomorphisms of grade $1 \widetilde{F}_{X, Y}: F X \otimes$ $F Y \rightarrow F(X \otimes Y)$, and an isomorphism of grade $1 F_{*}: I^{\prime} \rightarrow$ $F I$, such that, for all $X, Y, Z \in \mathrm{Ob} \mathbb{G}$, the following coherence conditions hold:

$$
\begin{gathered}
\widetilde{F}_{X, Y \otimes Z}\left(\operatorname{id}_{F X} \otimes \widetilde{F}_{Y, Z}\right) \mathbf{a}_{F X, F Y, F Z} \\
=F\left(\mathbf{a}_{X, Y, Z}\right) \widetilde{F}_{X \otimes Y, Z}\left(\widetilde{F}_{X, Y} \otimes \mathrm{id}_{F Z}\right), \\
F\left(\mathbf{r}_{X}\right) \widetilde{F}_{X, I}\left(\mathrm{id}_{F X} \otimes F_{*}\right)=\mathbf{r}_{F X}, \\
F\left(\mathbf{l}_{X}\right) \widetilde{F}_{I, X}\left(F_{*} \otimes \mathrm{id}_{F X}\right)=\mathbf{l}_{F X} .
\end{gathered}
$$

Let $\left(F, \widetilde{F}, F_{*}\right),\left(F^{\prime}, \widetilde{F}^{\prime}, F_{*}^{\prime}\right)$ be two $\Gamma$-graded monoidal functors. A graded monoidal natural equivalence $\theta: F \stackrel{\sim}{\rightarrow} F^{\prime}$ is a natural equivalence of functors such that all isomorphisms $\theta_{X}: F X \rightarrow F^{\prime} X$ are of grade 1 , and for all $X, Y \in \mathrm{Ob} \mathbb{G}$, the following coherence conditions hold:

$$
\widetilde{F}_{X, Y}^{\prime}\left(\theta_{X} \otimes \theta_{Y}\right)=\theta_{X \otimes Y} \widetilde{F}_{X, Y}, \quad \theta_{I} F_{*}=F_{*}^{\prime} .
$$

2.2. Reduced Graded Categorical Groups. The authors of [2] showed that any $\Gamma$-graded categorical group $\mathbb{G}=(\mathbb{G}, g r, \otimes, I$, a, $\mathbf{l}, \mathbf{r})$ determines a triple $(\Pi, A, h)$, where

(1) the set $\Pi=\pi_{0} \mathbb{G}$ of 1 -isomorphism classes of the objects in $\mathbb{G}$ is a $\Gamma$-group,

(2) the set $A=\pi_{1} \mathbb{G}$ of 1-automorphisms of the unit object $I$ is a $\Gamma$-equivariant $\Pi$-module,

(3) the third invariant is an equivariant cohomology class $[h] \in H_{\Gamma}^{3}(\Pi, A)$.

Based on these data, they constructed a $\Gamma$-graded categorical group, denoted by $\int_{\Gamma}(\Pi, A, h)$, which is graded monoidally equivalent to $\mathbb{G}$. Below, we briefly recall this construction.

Objects of $\int_{\Gamma}(\Pi, A, h)$ are elements $x \in \Pi$ and its morphisms are pairs $(a, \sigma): x \rightarrow y$ consisting of an element $a \in A$ and $\sigma \in \Gamma$ such that ${ }^{\sigma} x=y$.

The composition of two morphisms $(x \stackrel{(a, \sigma)}{\longrightarrow} y \stackrel{(b, \tau)}{\longrightarrow} z)$ is given by

$$
(b, \tau)(a, \sigma)=\left(b+{ }^{\tau} a+h(x, \tau, \sigma), \tau \sigma\right) .
$$

The graded tensor product $\int_{\Gamma}(\Pi, A, h) \times_{\Gamma} \int_{\Gamma}(\Pi, A, h) \rightarrow$ $\int_{\Gamma}(\Pi, A, h)$ is given by

$$
\begin{aligned}
(x & \stackrel{(a, \sigma)}{\longrightarrow} y) \otimes\left(x^{\prime} \stackrel{(b, \sigma)}{\longrightarrow} y^{\prime}\right) \\
& =\left(x x^{\prime} \stackrel{\left(a+y b+h\left(x, x^{\prime}, \sigma\right), \sigma\right)}{\longrightarrow} y y^{\prime}\right) .
\end{aligned}
$$

The unit constrains are strict in the sense that $\mathbf{l}_{x}=(0,1)=$ $\mathbf{r}_{x}: x \rightarrow x$. The associativity isomorphisms are

$$
\mathbf{a}_{x, y, z}=(h(x, y, z), 1):(x y) z \longrightarrow x(y z) .
$$

The stable $\Gamma$-grading is $\operatorname{gr}(a, \sigma)=\sigma$.

The unit graded functor $I: \Gamma \rightarrow \int_{\Gamma}(\Pi, A, h)$ is given by

$$
I(* \stackrel{\sigma}{\longrightarrow} *)=(1 \stackrel{(0, \sigma)}{\longrightarrow} 1) .
$$

We call the $\Gamma$-graded categorical group $\int_{\Gamma}(\Pi, A, h)$ a reduction of the $\Gamma$-graded categorical group $\mathbb{G}$, simply denoted by $S_{\mathbb{G}}$.

\section{Strict Graded Categorical Groups}

3.1. Definitions and Examples. It is well known that each crossed module of groups can be seen as a strict categorical group (see [6], Remark 3.1 in [7]). Crossed modules of groups can be enriched in some ways to become, for example, crossed bimodules over rings or equivariant crossed modules. In the former case, each crossed bimodule can be seen as a strict Ann-category [8]. In the later case, each crossed module of $\Gamma$-groups can be identified with a strict $\Gamma$-graded categorical group [5] whose definition is recalled as below.

Firstly, if $\left(F, \widetilde{F}, F_{*}\right)$ is a monoidal functor between categorical groups, then the isomorphism $F_{*}: I^{\prime} \rightarrow F I$ can be deduced from $F$ and $\widetilde{F}$, so we can omit $F_{*}$ when not necessary. A monoidal functor $(F, \widetilde{F}): \mathbb{G} \rightarrow \mathbb{G}^{\prime}$ between two categorical groups is termed regular whenever

$$
\begin{gathered}
F(x) \otimes F(y)=F(x \otimes y), \\
F(b) \otimes F(c)=F(b \otimes c),
\end{gathered}
$$

for all $x, y \in \mathrm{Ob} \mathbb{G}, b, c \in \operatorname{Mor} \mathbb{G}$. A factor set $(F, \theta)$ on $\Gamma$ with coefficients in a categorical group $\mathbb{G}$ (see [9]) is regular if $\theta^{\sigma, \tau}=\mathrm{id}$, and $F^{\sigma}$ is a regular monoidal functor, for all $\sigma, \tau \in \Gamma$.

Definition 1 (see [5]). A graded categorical group ( $\mathbb{G}, g r)$ is said to be strict if

(i) $\operatorname{Ker} \mathbb{G}$ is a strict categorical group,

(ii) $\mathbb{G}$ induces a regular factor set $(F, \theta)$ on $\Gamma$ with coefficients in a categorical group Ker $\mathbb{G}$.

Equivalently, a graded categorical group $(\mathbb{G}, g r)$ is strict if it is a $\Gamma$-graded extension of a strict categorical group by a regular factor set.

Note 1. In this paper we denote by + for the operation of the group $G$.

Example 2 (the strict graded categorical group $\mathrm{Dis}_{\Gamma} \Pi$ ). The discrete $\Gamma$-graded categorical group $\operatorname{Dis}_{\Gamma} \Pi$ defined by a $\Gamma$ group $\Pi$ has the elements of $\Pi$ as objects, and its morphisms $\sigma: x \rightarrow y$ are the elements $\sigma \in \Gamma$ with ${ }^{\sigma} x=y$. Composition is multiplication in $\Gamma$, and the grading $g r: \operatorname{Dis}_{\Gamma} \Pi \rightarrow \Gamma$ is the obvious map $\operatorname{gr}(\sigma)=\sigma$. The graded tensor product is given by

$$
(x \stackrel{\sigma}{\longrightarrow} y) \otimes\left(x^{\prime} \stackrel{\sigma}{\longrightarrow} y^{\prime}\right)=\left(x x^{\prime} \stackrel{\sigma}{\longrightarrow} y y^{\prime}\right)
$$


and the graded unit $I: \Gamma \rightarrow \operatorname{Dis}_{\Gamma} \Pi$ by

$$
I(* \stackrel{\sigma}{\longrightarrow} *)=(1 \stackrel{\sigma}{\longrightarrow} 1)
$$

the associativity and unit isomorphisms are identities.

Example 3 (the strict graded categorical group $\mathrm{Hol}_{\Gamma} G$ of a $\Gamma$-group $G$ ). Firstly, observe that if $G$ is a $\Gamma$-group, then the group Aut $G$ of automorphisms of $G$ is also a $\Gamma$-group with the action

$$
\left({ }^{\sigma} f\right)(b)={ }^{\sigma}\left(f\left({ }^{\sigma^{-1}} b\right)\right), \quad b \in G, f \in \operatorname{Aut} G .
$$

Then, the homomorphism $\mu: G \rightarrow$ Aut $G, b \mapsto \mu_{b}\left(\mu_{b}\right.$ is the inner automorphism of $G$ given by conjugation with $b$ ) is a homomorphism of $\Gamma$-groups. Indeed, for all $\sigma \in \Gamma, a, b \in G$ one has

$$
\begin{aligned}
\mu_{b}(a) & ={ }^{\sigma} b+a-{ }^{\sigma} b \\
& ={ }^{\sigma}\left(b+{ }^{\sigma^{-1}} a-b\right) \\
& ={ }^{\sigma}\left(\mu_{b}\left({ }^{\sigma^{-1}} a\right)\right)=\left({ }^{\sigma} \mu_{b}\right)(a) .
\end{aligned}
$$

For each $\Gamma$-group $G$, we can construct a strict graded categorical group, denoted by $\mathrm{Hol}_{\Gamma} G$ (see [2]), whose objects are elements of the $\Gamma$-group Aut $G$. A $\sigma$-morphism $\alpha \rightarrow \beta$ is a pair $(u, \sigma)$, where $u \in G$ with ${ }^{\sigma} \alpha=\mu_{u} \beta$. Composition of two morphisms $\alpha \stackrel{(u, \sigma)}{\longrightarrow} \beta \stackrel{(v, \tau)}{\longrightarrow} \gamma$ is given by

$$
(v, \tau) \circ(u, \sigma)=\left({ }^{\tau} u+v, \tau \sigma\right) .
$$

The graded tensor product is

$$
(\alpha \stackrel{(u, \sigma)}{\longrightarrow} \beta) \otimes\left(\alpha^{\prime} \stackrel{\left(u^{\prime}, \sigma\right)}{\longrightarrow} \beta^{\prime}\right)=\left(\alpha \alpha^{\prime} \stackrel{\left(u+\beta\left(u^{\prime}\right), \sigma\right)}{\longrightarrow} \beta \beta^{\prime}\right),
$$

and the graded unit $I: \Gamma \rightarrow \mathrm{Hol}_{\Gamma} G$ is defined by

$$
I(* \stackrel{\sigma}{\longrightarrow} *)=\mathrm{id}_{G} \stackrel{(0, \sigma)}{\longrightarrow} \mathrm{id}_{G} .
$$

The associativity and unit isomorphisms are identities.

Example 4 (strict graded categorical groups associated with an equivariant crossed module). The notion of $\Gamma$-crossed module is a generalization of that of crossed module of groups introduced by Whitehead [10].

Definition 5 (see [5]). Let $B, D$ be $\Gamma$-groups. A $\Gamma$-crossed module is a quadruple $(B, D, d, \vartheta)$, where $d: B \rightarrow D, \vartheta$ : $D \rightarrow$ Aut $B$ are $\Gamma$-homomorphisms satisfying the following conditions:

$$
\begin{gathered}
C_{1} \cdot \vartheta d=\mu, \\
C_{2} \cdot d\left(\vartheta_{x}(b)\right)=\mu_{x}(d(b)), \\
C_{3} \cdot{ }^{\sigma}\left(\vartheta_{x}(b)\right)=\vartheta_{\left({ }^{\sigma} x\right)}\left({ }^{\sigma} b\right),
\end{gathered}
$$

where $\sigma \in \Gamma, x \in D, b \in B$, and $\mu_{x}$ is the inner automorphism given by conjugation with $x$.
A $\Gamma$-crossed module is also called an equivariant crossed module by Noohi [11].

From the definition of $\Gamma$-crossed module, it is easy to deduce the following properties: (i) Ker $d$ is a $\Gamma$-subgroup in $Z(B)$; (ii) Im $d$ is both a normal subgroup in $D$ and a $\Gamma$-group; (iii) Ker $d$ is a left $\Gamma$-equivariant Coker $d$-module under the actions

$$
s a=\varphi_{x}(a), \quad{ }^{\sigma} s=\left[{ }^{\sigma} x\right], \quad a \in \operatorname{Ker} d, x \in s \in \text { Coker } d .
$$

The strict graded categorical group $\mathbb{G}$ associated with the $\Gamma$-crossed module $(B, D, d, \vartheta)$ is constructed as follows.

Objects of $\mathbb{G}$ are the elements of the group $D$, and a $\sigma$ morphism $x \rightarrow y$ is a pair $(b, \sigma)$, where $b \in B, \sigma \in \Gamma$ with ${ }^{\sigma} x=d(b) y$. Composition of two morphisms is defined by

$$
(x \stackrel{(b, \sigma)}{\longrightarrow} y \stackrel{(c, \tau)}{\longrightarrow} z)=\left(x \stackrel{\left({ }^{\tau} b+c, \tau \sigma\right)}{\longrightarrow} z\right) .
$$

The tensor product on objects is given by the multiplication in the group $D$, and for two morphisms $(x \stackrel{(b, \sigma)}{\longrightarrow} y)$, $\left(x^{\prime} \stackrel{(c, \sigma)}{\longrightarrow} y^{\prime}\right)$, then

$$
(x \stackrel{(b, \sigma)}{\longrightarrow} y) \otimes\left(x^{\prime} \stackrel{(c, \sigma)}{\longrightarrow} y^{\prime}\right)=\left(x x^{\prime} \stackrel{\left(b+\vartheta_{y} c, \sigma\right)}{\longrightarrow} y y^{\prime}\right) .
$$

The associativity and unit constraints of the tensor product are strict.

The graded functor $\mathrm{gr}: \mathbb{G} \rightarrow \Gamma$ is given by

$$
(b, \sigma) \longmapsto \sigma
$$

and the graded unit functor $I: \Gamma \rightarrow \mathbb{G}$ by

$$
I(* \stackrel{\sigma}{\longrightarrow} *)=(1 \stackrel{(0, \sigma)}{\longrightarrow} 1) .
$$

3.2. Equivariant Kernel $p: \Pi \rightarrow$ Out $G$ and Strict $\Gamma$-Categorical Group $\operatorname{Hol}_{\Gamma}(G)$. The notion of equivariant kernel was introduced in [2]. It is a triple $(\Pi, G, p)$, where $\Pi, G$ are $\Gamma$ groups and $p: \Pi \rightarrow$ Aut $G / \operatorname{In} G$ is a homomorphism of $\Gamma$-groups. Then $Z G$ is $\Gamma$-equivariant Out $G$-module under the action $[f] a=f(a)$. The obstruction of $(\Pi, G, p)$ is an element $\operatorname{Obs}(p) \in H_{\Gamma}^{3}(\Pi, Z G)$, defined as follows (see [2, page 996]). For each $x \in \Pi$, choose an automorphism $\varphi_{x}$ of $G, \varphi_{x} \in p(x)$, in particular $\varphi_{1}=\mathrm{id}_{G}$. Since

$$
\begin{array}{r}
p(x y)=p(x) p(y), \quad{ }^{\sigma} p(x)=p\left({ }^{\sigma} x\right), \\
x, y \in \Pi, \quad \sigma \in \Gamma,
\end{array}
$$

there exist elements $f(x, y), f(x, \sigma) \in G$ such that

$$
\begin{gathered}
\varphi_{x} \varphi_{y}=\mu_{f(x, y)} \varphi_{x y}, \\
{ }^{\sigma} \varphi_{x}=\mu_{f(x, \sigma)} \varphi_{\left({ }^{\sigma} x\right)},
\end{gathered}
$$

where $\mu_{u}$ is the inner automorphism of $\Gamma$-group $G$ induced by $u \in G$. The pair $(f, \varphi)$ therefore induces an element 
$k \in Z_{\Gamma}^{3}(\Pi, Z G)$ defined by the following relations. The associative law for a product $\varphi_{x} \varphi_{y} \varphi_{z}$ leads to

$$
\begin{aligned}
\varphi_{x} f & (y, z)+f(x, y z) \\
& =k(x, y, z)+f(x, y)+f(x y, z) .
\end{aligned}
$$

The relations

$$
{ }^{\sigma}\left(\varphi_{x} \varphi_{y}\right)={ }^{\sigma} \varphi_{x}^{\sigma} \varphi_{y}, \quad{ }^{\tau}\left({ }^{\sigma} \varphi_{x}\right)={ }^{\tau \sigma} \varphi_{x}
$$

imply

$$
\begin{array}{r}
f(x, \sigma)+\varphi_{\left({ }^{\sigma} x\right)}(f(y, \sigma))+f\left({ }^{\sigma} x,{ }^{\sigma} y\right) \\
=k(x, y, \sigma)+{ }^{\sigma} f(x, y)+f(x y, \sigma) \\
{ }^{\tau} f(x, \sigma)+f\left({ }^{\sigma} x, \tau\right)=k(x, \tau, \sigma)+f(x, \tau \sigma)
\end{array}
$$

According to [2], $k \in Z_{\Gamma}^{3}(\Pi, Z G)$. The cohomology class [ $\left.k\right]$ is called the obstruction of $p$, denoted by $\operatorname{Obs}(p)$.

Theorem 4.1 in [2] states that equivariant extensions $G$ by $\Pi$ inducing $p$ exist if and only if $\operatorname{Obs}(p)=0$. By Theorem 4.2 in [2], there is a bijection

$$
\operatorname{Ext}_{\Gamma}(\Pi, G) \simeq \operatorname{Hom}\left[\operatorname{Dis}_{\Gamma} \Pi, \operatorname{Hol}_{\Gamma} G\right] .
$$

The following theorem describes the invariants of the graded categorical group $\mathrm{Hol}_{\Gamma} G$ and shows a relation between third invariant of $\operatorname{Hol}_{\Gamma} G$ and $\operatorname{Obs}(p)$.

Theorem 6. Let $(\Pi, G, p)$ be an equivariant kernel. Then the invariants of the strict graded categorical group $\mathrm{Hol}_{\Gamma} G$ are

(i) $\pi_{0}\left(\operatorname{Hol}_{\Gamma} G\right)=\operatorname{Out} G, \pi_{1}\left(\operatorname{Hol}_{\Gamma} G\right)=Z G$,

(ii) $h \in Z_{\Gamma}^{3}(\operatorname{Out} G, Z G)$ with $p^{*} h \in \operatorname{Obs}(p)$.

Proof. (i) It is obvious.

(ii) According to Section 3 in [2], there is a $\Gamma$-monoidal equivalence

$$
(H, \widetilde{H}): \int_{\Gamma}(\operatorname{Out} G, Z G, h) \longrightarrow \operatorname{Hol}_{\Gamma} G .
$$

Let us observe that each morphism $(a, \sigma): r \rightarrow s$ in $\int_{\Gamma}($ Out $G, Z G, h)$ is written in the form

$$
r \stackrel{(0, \sigma)}{\longrightarrow} \sigma \stackrel{(a, 1)}{\longrightarrow} s .
$$

Let

$$
H\left(r \stackrel{(0, \sigma)}{\longrightarrow}^{\sigma} r\right)=\left(H(r) \stackrel{(g(r, \sigma), \sigma)}{\longrightarrow}^{\sigma} H(r)\right) .
$$

Since $H(r \stackrel{(a, 1)}{\longrightarrow} r)=(a, 1)$, for $r \in$ Out $G, a \in Z G$ (see [12, Proposition 13]), the $\gamma$-monoidal functor $(H, \widetilde{H})$ defines a function $f$ by

$$
\begin{aligned}
& f(x, y)=\widetilde{H}_{p x, p y}=\left(p^{*} \widetilde{H}\right)(x, y), \\
& f(x, \sigma)=g(p x, \sigma)=\left(p^{*} g\right)(x, \sigma) .
\end{aligned}
$$

Set $\varphi=H \circ p: \Pi \rightarrow$ Out $G \rightarrow$ Aut $G$, and the pair $(f, \varphi)$ determines a function $k \in \operatorname{Obs}(p)$ by relations (25)-(28) which can be written in the form $k=\delta f$, even though $f$ does not take values in $Z G$.

The compatibility of $(H, \widetilde{H})$ with the associativity constraints leads to $h=\delta \widetilde{H}$. Thus, for $x, y, z \in \Pi$, we obtain

$$
\begin{aligned}
\left(p^{*} h\right)(x, y, z) & =h(p x, p y, p z) \\
& =(\delta \widetilde{H})(p x, p y, p z) \\
& =(\delta f)(x, y, z) \stackrel{(25)}{=} k(x, y, z) .
\end{aligned}
$$

Next, we show that $\left(p^{*} h\right)(x, y, \sigma)=k(x, y, \sigma)$.

Since $(H, \widetilde{H})$ is a $\otimes$-functor, the following diagram commutes:

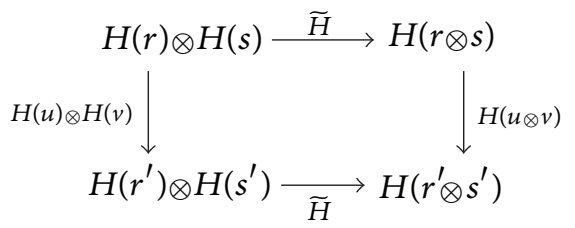

where $u=\left(r \stackrel{(0, \sigma)}{\longrightarrow} r^{\prime}\right), v=\left(s \stackrel{(0, \sigma)}{\longrightarrow} s^{\prime}\right)$.

Due to the relations (5)-(15) of the $\otimes$ product and the composition in $\int_{\Gamma}($ Out $G, Z G, h)$ and $\operatorname{Hol}_{\Gamma} G$, one has

$$
\begin{gathered}
H(u \otimes v) \circ \widetilde{H}_{r, s}=\left({ }^{\sigma} H_{r, s}+g(r s, \sigma)+h(r, s, \sigma), \sigma\right), \\
\widetilde{H}_{r^{\prime}, s^{\prime}} \circ(H(u) \otimes H(v)) \\
=\left(g(r, \sigma)+H\left(r^{\prime}\right) g\left(s^{\prime}, \sigma\right)+\widetilde{H}_{r^{\prime}, s^{\prime}}, \sigma\right) .
\end{gathered}
$$

Let us note that

$$
\begin{gathered}
H_{r, s}=H_{p x, p y}=f(x, y), \\
h(r, s, \sigma)=\left(p^{*} h\right)(x, y, \sigma), \\
g(r, \sigma)=g(p(x), \sigma)=\left(p^{*} g\right)(x, \sigma)=f(x, \sigma), \\
H\left(r^{\prime}\right)=H\left({ }^{\sigma} r\right)={ }^{\sigma} H(r)={ }^{\sigma} H(p(x))={ }^{\sigma} \varphi_{x} .
\end{gathered}
$$

Therefore,

$$
\begin{aligned}
& { }^{\sigma} f(x, y)+f(x y, \sigma)+\left(p^{*} h\right)(x, y, \sigma) \\
& =f(x, \sigma)+{ }^{\sigma} \varphi_{x} f(y, \sigma)+f\left({ }^{\sigma} x,{ }^{\sigma} y\right) .
\end{aligned}
$$

This shows that $\left(p^{*} h\right)(x, y, \sigma) \stackrel{(27)}{=} k(x, y, \sigma)$.

Finally, we prove that $\left(p^{*} h\right)(x, \tau, \sigma)=k(x, \tau, \sigma)$ based on the functorial property of $H$. Indeed, consider the composition

$$
r \stackrel{(0, \sigma)}{\longrightarrow} s \stackrel{(0, \tau)}{\longrightarrow} t .
$$


On one hand,

$$
\begin{aligned}
H & {[(0, \tau) \circ(0, \sigma)] } \\
& \stackrel{(5)}{=} H[(h(r, \tau, \sigma), \tau \sigma)]=H[h(r, \tau, \sigma), 1) \circ(0, \tau \sigma)] \\
& =H[(h(r, \tau, \sigma), 1)] \circ H[(0, \tau \sigma)] \\
& =(h(r, \tau, \sigma), 1) \circ(g(r, \tau \sigma), \tau \sigma) \\
& \stackrel{(14)}{=}(g(r, \tau \sigma)+h(r, \tau, \sigma), \tau \sigma) .
\end{aligned}
$$

On the other hand,

$$
\begin{aligned}
H & (0, \tau) \circ H(0, \sigma) \\
& =H(r) \stackrel{(g(r, \sigma), \sigma)}{\longrightarrow} H(s) \stackrel{(g(s, \tau), \tau)}{\longrightarrow} H(t) \\
& \stackrel{(14)}{=} H(r) \stackrel{\left({ }^{\tau} g(r, \sigma)+g\left({ }^{\sigma} r, \tau\right), \tau \sigma\right)}{\longrightarrow} H(t) .
\end{aligned}
$$

Again, $h(r, \tau, \sigma) \in Z G$, the functorial property of $H$, implies

$$
h(r, \tau, \sigma)+g(r, \tau \sigma)={ }^{\tau} g(r, \sigma)+g\left({ }^{\sigma} r, \tau\right),
$$

or $h(r, \tau, \sigma)=\delta g$. Clearly,

$$
\begin{aligned}
\left(p^{*} h\right)(x, \tau, \sigma) & =h(p x, \tau, \sigma)=\delta g(p x, \tau, \sigma) \\
& =\delta f(x, \tau, \sigma) \stackrel{(28)}{=} k(x, \tau, \sigma) .
\end{aligned}
$$

3.3. Classification of Central Extensions. Denote by $\operatorname{Ext}_{\Gamma}^{c}(\Pi$, $A$ ) the set of all equivalence classes of equivariant extensions $A \nrightarrow E \rightarrow \Pi$, where $A \subset Z E$. One can classify these extensions by the autofunctors of $\Gamma$-graded categorical group $\int_{\Gamma}(\Pi, A, 0)$.

Theorem 7 (the schreier theory for central extensions of equivariant groups). Let $\Pi$ be a $\Gamma$-group, and let $A$ be a $\Gamma$ equivariant $\Pi$-module. There is a bijection

$$
\operatorname{Ext}_{\Gamma}^{c}(\Pi, A) \simeq \operatorname{End}_{\Gamma}^{\text {id }}\left(\int_{\Gamma}(\Pi, A, 0)\right),
$$

where $\mathrm{End}_{\Gamma}^{\mathrm{id}}$ is the set of homotopy classes of monoidal $\Gamma$-functors $F$ from $\int_{\Gamma}(\Pi, A, 0)$ to itself satisfying

$$
\begin{aligned}
& F(x)=x, \quad x \in \Pi, \\
& F(b, 1)=(b, 1), \quad b \in A .
\end{aligned}
$$

Proof. Let $(F, \widetilde{F}) \in \operatorname{End}_{\Gamma}^{\text {id }}\left(\int_{\Gamma}(\Pi, A, 0)\right)$. Then $(F, \widetilde{F})$ defines a function

$$
\varphi:(\Pi \times \Pi) \cup(\Pi \times \Gamma) \longrightarrow A
$$

by

$$
(\varphi(x, y), 1)=\widetilde{F}_{x, y}, \quad(\varphi(x, \sigma), \sigma)=F\left(x \stackrel{(0, \sigma)}{\longrightarrow}^{\sigma} x\right),
$$

with $\varphi\left(x, 1_{\Gamma}\right)=0$. The compatibilities of $(F, \widetilde{F})$ with the constraints imply $\varphi(x, 1)=0=\varphi(1, y)$ and

$$
\varphi(x, y)+\varphi(x y, z)=x(\varphi(y, z))+\varphi(x, y z) .
$$

The naturalness of $\widetilde{F}$ and the functority of $F$ give, respectively,

$$
\begin{gathered}
{ }^{\sigma} \varphi(x, y)+\varphi(x y, \sigma) \\
=\varphi(x, \sigma)+\left({ }^{\sigma} x\right) \varphi(y, \sigma)+\varphi\left({ }^{\sigma} x,{ }^{\sigma} y\right), \\
\varphi(x, \tau \sigma)={ }^{\sigma} \varphi(x, \tau)+\varphi\left({ }^{\tau} x, \sigma\right),
\end{gathered}
$$

for all $x, y, z \in G, \sigma, \tau \in \Gamma$. With the function $\varphi$, we can construct an equivariant crossed product extension $E_{\varphi}=$ $A \times_{\varphi} \Pi$ in which the operation of $E_{\varphi}$ is

$$
(a, x)+(b, y)=(a+x b+\varphi(x, y), x y) .
$$

The relation (48) and the normality of $\varphi$ show that $E_{\varphi}$ is a group. By the relations (49), (50), $E_{\varphi}$ is a $\Gamma$-group under the $\Gamma$-action

$$
{ }^{\sigma}(a, x)=\left({ }^{\sigma} a+\varphi(x, \sigma),{ }^{\sigma} x\right) .
$$

Then we obtain an exact sequence

$$
0 \longrightarrow A \stackrel{i}{\longrightarrow} E_{\varphi} \stackrel{q}{\longrightarrow} \Pi \longrightarrow 1,
$$

where $i(a)=(a, 1), q(a, x)=x$. Further, it is easy to see that $A \subset Z\left(E_{\varphi}\right)$.

Conversely, suppose that

$$
0 \longrightarrow A \stackrel{i}{\longrightarrow} E \stackrel{q}{\longrightarrow} \Pi \longrightarrow 1
$$

is an equivariant extension with $A \subset Z(E)$. For each $x \in \Pi$, choose a representative $u_{x} \in E, u_{1}=(0)$. Then the system $\left\{u_{x} \mid x \in \Pi\right\}$ induces a function $\varphi$ taking values in $A$ such that

$$
\begin{gathered}
u_{x}+u_{y}=\varphi(x, y)+u_{x y}, \\
{ }^{\sigma} u_{x}=\varphi(x, \sigma)+u_{\sigma_{x}} .
\end{gathered}
$$

The rules

$$
\begin{gathered}
u_{x}+\left(u_{y}+u_{z}\right)=\left(u_{x}+u_{y}\right)+u_{z}, \\
{ }^{\sigma}\left(u_{x}+u_{y}\right)={ }^{\sigma}\left(u_{x}\right)+{ }^{\sigma}\left(u_{y}\right), \quad{ }^{\tau}\left({ }^{\sigma} u_{x}\right)={ }^{\tau \sigma} u_{x}
\end{gathered}
$$

imply the relations (48)-(50), respectively. Thus, we can define a $\Gamma$-monoidal functor $(F, \widetilde{F}) \in \operatorname{End}_{\Gamma}\left(\int_{\Gamma}(\Pi, A, 0)\right)$ as follows:

$$
\begin{gathered}
F x=x, \quad F(b, 1)=(b, 1), \\
F\left(x \stackrel{(0, \sigma)}{\longrightarrow}^{\sigma} x\right)=(\varphi(x, \sigma), \sigma), \quad \widetilde{F}_{x, y}=(\varphi(x, y), 1) .
\end{gathered}
$$

Now, two extensions in $\operatorname{Ext}_{\Gamma}^{c}(\Pi, A)$ are equivalent if and only if two corresponding graded monoidal functors are 
homotopic. Indeed, let $F, F^{\prime}: \int_{\Gamma}(\Pi, A, 0) \rightarrow \int_{\Gamma}(\Pi, A, 0)$ be two homotopic $\Gamma$-graded monoidal functors in which the homotopy $\varepsilon: F \rightarrow F^{\prime}$ is given by

$$
\varepsilon_{x}=(x \stackrel{(e(x), 1)}{\longrightarrow} x), \quad x \in \Pi,
$$

where $e: \Pi \rightarrow A$. From the definition of a homotopy and from the relations (5), (6), it follows that

$$
\begin{gathered}
e(1)=0, \\
\varphi(x, \sigma)+e\left({ }^{\sigma} x\right)={ }^{\sigma} e(x)+\varphi^{\prime}(x, \sigma), \\
\varphi(x, y)+e(x y)=e(x)+x e(y)+\varphi^{\prime}(x, y),
\end{gathered}
$$

where $x, y \in \Pi, \sigma \in \Gamma$. Then, the corresponding

$$
\begin{gathered}
\beta: E_{\varphi} \longrightarrow E_{\varphi^{\prime}} \\
(a, x) \longmapsto(a+e(x), x)
\end{gathered}
$$

is an equivalence between two central extensions if and only if equalities (59) hold.

Conversely, if $\beta: E_{\varphi} \rightarrow E_{\varphi^{\prime}}$ is an isomorphism, then

$$
\beta(a, x)=(a+e(x), x),
$$

where $e: \Pi \rightarrow A$ is a function with $e(1)=0$. Thus, it follows that $\varepsilon_{x}=(e(x), 1)$ is a homotopy of $F$ and $F^{\prime}$, as we see by retracting our steps.

Finally, each central extension $E$ is equivalent to one $E_{\varphi}$ via the isomorphism $\alpha: a+u_{x} \mapsto(a, x)$.

3.4. Composition of a $\Gamma$-Categorical Group with a $\Gamma$-Homomorphism. It is well known that for a given extension

$$
E: 0 \longrightarrow A \stackrel{i}{\longrightarrow} B \stackrel{q}{\longrightarrow} \Pi \rightarrow 1,
$$

where $A$ is Abelian, and a given homomorphism $\gamma: \Pi^{\prime} \rightarrow$ $\Pi$, there is an extension $E^{\prime}$ of $A$ by $\Pi^{\prime}$ with $E^{\prime}=E \gamma$. The extension $E^{\prime}$ is just the pull pack of the pair $(q, \gamma)$. We state an analogous result on strict $\Gamma$-categorical groups in the following proposition.

Proposition 8. Let $\mathbb{W}$ be a strict graded categorical group with three invariants $\Pi, C, h$, and let $p: \Pi^{\prime} \rightarrow \Pi$ be an equivariant homomorphism. Then there exists a strict graded categorical group $\mathbb{G}$ which is equivalent to the graded categorical group $\int_{\Gamma}\left(\Pi^{\prime}, C, h^{\prime}\right)$, where $C$ is viewed as a $G^{\prime}$-module under the actions $x c=p(x) c, x \in \Pi^{\prime}, c \in C$, and $\left[h^{\prime}\right]=\left[p^{*} h\right]$.

Proof. The strict graded categorical group $\mathbb{G}$ is constructed as follows:

$$
\mathrm{Ob} \mathbb{G}=\left\{(x, X) \mid x \in \Pi^{\prime}, X \in p(x)\right\} .
$$

A $\sigma$-morphism $(x, X) \rightarrow(x, Y)$ is a triple $(x, u, \sigma)$, where $u: X \rightarrow Y$ is a $\sigma$-morphism in $\mathbb{H}$. The composition of two morphisms $(x, X) \stackrel{(x, u, \sigma)}{\longrightarrow}(x, Y) \stackrel{(x, v, \tau)}{\longrightarrow}(x, Z)$ is given by

$$
(x, v, \tau) \circ(x, u, \sigma)=(x, v \circ u, \tau \sigma) .
$$

The tensor products on objects and on morphisms in $\mathbb{G}$ are given by

$$
\begin{gathered}
(x, X) \otimes(y, Y)=(x y, X \otimes Y), \\
(x, u, \sigma) \otimes(y, v, \sigma)=(x y, u \otimes v, \sigma) .
\end{gathered}
$$

For each morphism $(x, u, \sigma)$ in $\mathbb{G}$, one has

$$
(x, u, \sigma)^{-1}=\left(x, u^{-1}, \sigma^{-1}\right) .
$$

The graded functor $\mathrm{gr}: \mathbb{G} \rightarrow \Gamma$ is given by

$$
(x, u, \sigma) \longmapsto \sigma
$$

and the graded unit functor $I: \Gamma \rightarrow \mathbb{G}$ by

$$
\sigma \longmapsto\left((1, I) \stackrel{\left(1, \mathrm{id}_{I}, \sigma\right)}{\longrightarrow}(1, I)\right)
$$

The unit object of $\mathbb{G}$ is $(1, I)$, where $I$ is the unit object of $\mathbb{H}$. The associativity and unit constraints are identities.

Suppose that the graded categorical group $\mathbb{H}$ induces a regular factor set $\left\{F_{\mathbb{a}}^{\sigma}, \sigma \in \Gamma\right\}$. Then, $\mathbb{G}$ induces a regular factor set $\left\{F_{\mathbb{G}}^{\sigma}, \sigma \in \Gamma\right\}$ by

$$
\begin{gathered}
F_{\mathbb{G}}^{\sigma}(x, X)=\left(x, F_{\mathbb{\square}}^{\sigma} X\right), \\
F_{\mathbb{G}}^{\sigma}(x, u, \tau)=\left(x, F_{\mathbb{\square}}^{\sigma}(u), \tau\right) .
\end{gathered}
$$

Thus, $\mathbb{G}$ is a strict graded categorical group.

We define a pair $(\varphi, f)$ of maps by

$$
\begin{array}{cl}
\lambda: \pi_{0} \mathbb{G} \longrightarrow \Pi^{\prime}, & f: \pi_{1} \mathbb{G} \longrightarrow \pi_{1} \mathbb{W}=C, \\
{[(x, X)] \longmapsto x,} & (1, c, \sigma) \longmapsto(c, \sigma) .
\end{array}
$$

Then, $\lambda$ is an isomorphism of $\Gamma$-groups and $f$ is an isomorphism of $\Gamma$-equivariant $\Pi^{\prime}$-module, where the $\Pi^{\prime}$-module structure of $C$ is $x c=p(x) c, x \in \Pi^{\prime}, c \in C$.

The graded monoidal functor $(F, \widetilde{F}): \mathbb{G} \rightarrow \mathbb{M}$ given by

$$
F(x, X)=X, \quad F(x, u, \sigma)=(u, \sigma), \quad \widetilde{F}=\mathrm{id}
$$

induces one $S_{F}=(\phi, \widetilde{\phi}): S_{\mathbb{G}} \rightarrow S_{\mathbb{\square}}$, where $S_{\mathbb{\square}}=\int_{\Gamma}(\Pi, C, h)$. Then,

$$
\begin{aligned}
\phi[(x, X)] & =F_{0}[(x, X)]=[F(x, X)] \\
& =[X]=p(x)=p \lambda[(x, X)], \\
\phi(1, c, \sigma) & =F_{1}(1, c, \sigma)=\gamma_{F(1, I)}^{-1} F(1, c, \sigma) \\
& =\gamma_{I}^{-1}(c, \sigma)=(c, \sigma)=f(1, c, \sigma),
\end{aligned}
$$

where $(c, \sigma)$ is a morphism in $S_{\mathbb{\square}}$. Thus, $S_{F}$ is a functor of type $(p \lambda, f)$.

Suppose that $h_{\mathbb{G}} \in Z_{\Gamma}^{3}\left(\pi_{0} \mathbb{G}, \pi_{1} \mathbb{G}\right)$. According to Theorem 3.2 in [2], the obstruction of pair $(p \lambda, f)$ vanishes in $H^{3}\left(\pi_{0} \mathbb{G}, \pi_{1} \mathbb{M}\right)=H^{3}\left(\pi_{0} \mathbb{G}, C\right)$; that is,

$$
(p \lambda)^{*} h=f_{*} h_{\mathbb{G}}+\delta \tilde{\phi} .
$$


Now, we denote $h^{\prime}=f_{*} h_{\mathbb{G}}$, and then the pair $(J, \widetilde{J})$, where $J=(\lambda, f), \widetilde{J}=\mathrm{id}$, is a graded monoidal functor from $S_{\mathbb{G}}$ to $\mathbb{J}=\int_{\Gamma}\left(\Pi^{\prime}, C, h^{\prime}\right)$. The composition

$$
\mathbb{G} \stackrel{(G, \widetilde{G})}{\longrightarrow} S_{\mathbb{G}} \stackrel{(J, \widetilde{J})}{\longrightarrow} \mathbb{J}
$$

is an equivalence from $\mathbb{G}$ to $\mathbb{J}$.

Finally, we prove that $h^{\prime}$ is in the same cohomology class with $p^{*} h$. Let $K=\left(\lambda^{-1}, f^{-1}\right): \mathbb{J} \rightarrow S_{\mathbb{G}}$; then $K$ together with $\widetilde{K}=\mathrm{id}$ is a graded monoidal functor, and the composition

$$
(\phi, \widetilde{\phi}) \circ(K, \widetilde{K}): \mathbb{} \longrightarrow S_{\mathbb{\sharp}}
$$

is a graded monoidal functor making the following diagram commute:

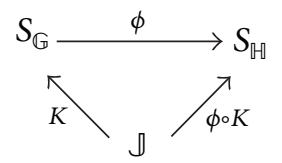

Since $\phi \circ K$ is a graded monoidal functor of type ( $p$,id), its obstruction vanishes. This implies that $p^{*} h-h^{\prime}=\partial g$, and that means that $\left[h^{\prime}\right]=\left[p^{*} h\right]$.

If $\Gamma=1$, we obtain Proposition 14 in [12].

The graded categorical group $\mathbb{G}$ is called a composition of $\mathbb{U}$ with $p$, denoted by $\mathbb{G}=\mathbb{U} \circ p$. It follows that $\mathbb{G}$ can be seen as a contravariant functor in terms of the invariant $\pi_{0} \mathbb{G}$ with $\pi_{1} \mathbb{G}$ fixed. Indeed,

$$
\mathbb{G} \circ \mathrm{id}_{\pi_{0} \mathbb{G}}=\mathbb{G}, \quad \mathbb{G} \circ\left(p \circ p^{\prime}\right)=(\mathbb{G} \circ p) \circ p^{\prime} .
$$

\section{References}

[1] S. M. Lane, Homology, Springer, Berlin, Germany, 1975.

[2] A. M. Cegarra, J. M. García-Calcines, and J. A. Ortega, "On graded categorical groups and equivariant group extensions," Canadian Journal of Mathematics, vol. 54, no. 5, pp. 970-997, 2002.

[3] H. X. Sinh, Gr-catégories [Thèse de doctorat], Université Paris VII, Paris, France, 1975.

[4] A. Fröhlich and C. T. C. Wall, "Graded monoidal categories," Compositio Mathematica, vol. 28, pp. 229-285, 1974.

[5] N. T. Quang and P. T. Cuc, "Equivariant crossed modules and Cohomology of group with operators," http://arxiv.org/abs/1302 .4573 .

[6] R. Brown and C. B. Spencer, "G-groupoids, crossed modules and the fundamental groupoid of a topological group," vol. 38, no. 4, pp. 296-302, 1976.

[7] A. Joyal and R. Street, "Braided tensor categories," Advances in Mathematics, vol. 102, no. 1, pp. 20-78, 1993.

[8] N. T. Quang and P. T. Cuc, "Crossed bimodules over rings and Shukla cohomology," Mathematical Communications, vol. 17, no. 2, pp. 575-598, 2012.

[9] A. M. Cegarra, A. R. Garzón, and J. A. Ortega, "Graded extensions of monoidal categories," Journal of Algebra, vol. 241, no. 2, pp. 620-657, 2001.
[10] J. H. C. Whitehead, “Combinatorial homotopy II," Bulletin of the American Mathematical Society, vol. 55, pp. 453-496, 1949.

[11] B. Noohi, "Group cohomology with coefficients in a crossed module," Journal of the Institute of Mathematics of Jussieu, vol. 10, no. 2, pp. 359-404, 2011.

[12] N. T. Quang, N. T. Thuy, and P. T. Cuc, "Monoidal functors between (braided) GR-categories and their applications," EastWest Journal of Mathematics, vol. 13, no. 2, pp. 170-193, 2011. 


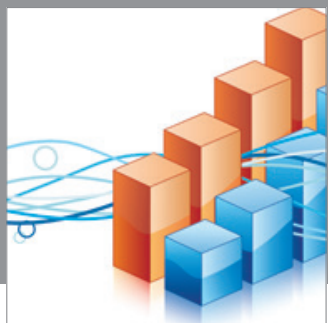

Advances in

Operations Research

mansans

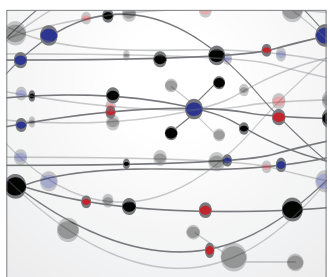

The Scientific World Journal
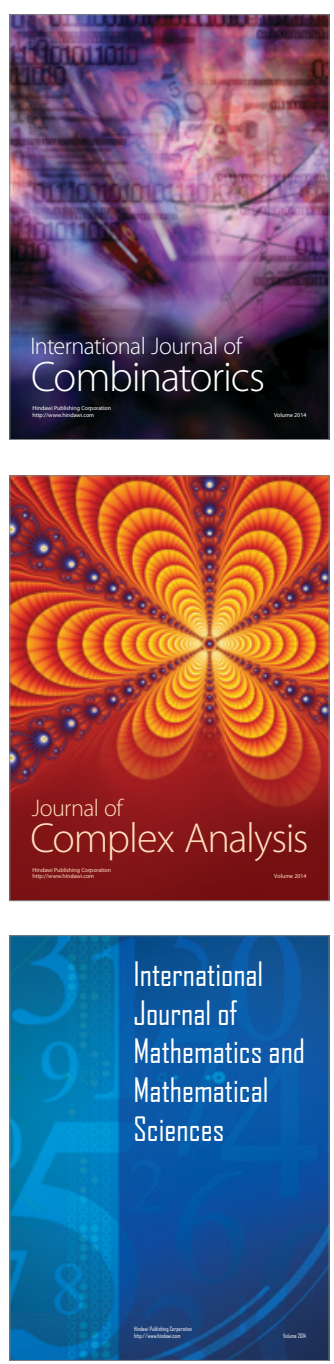
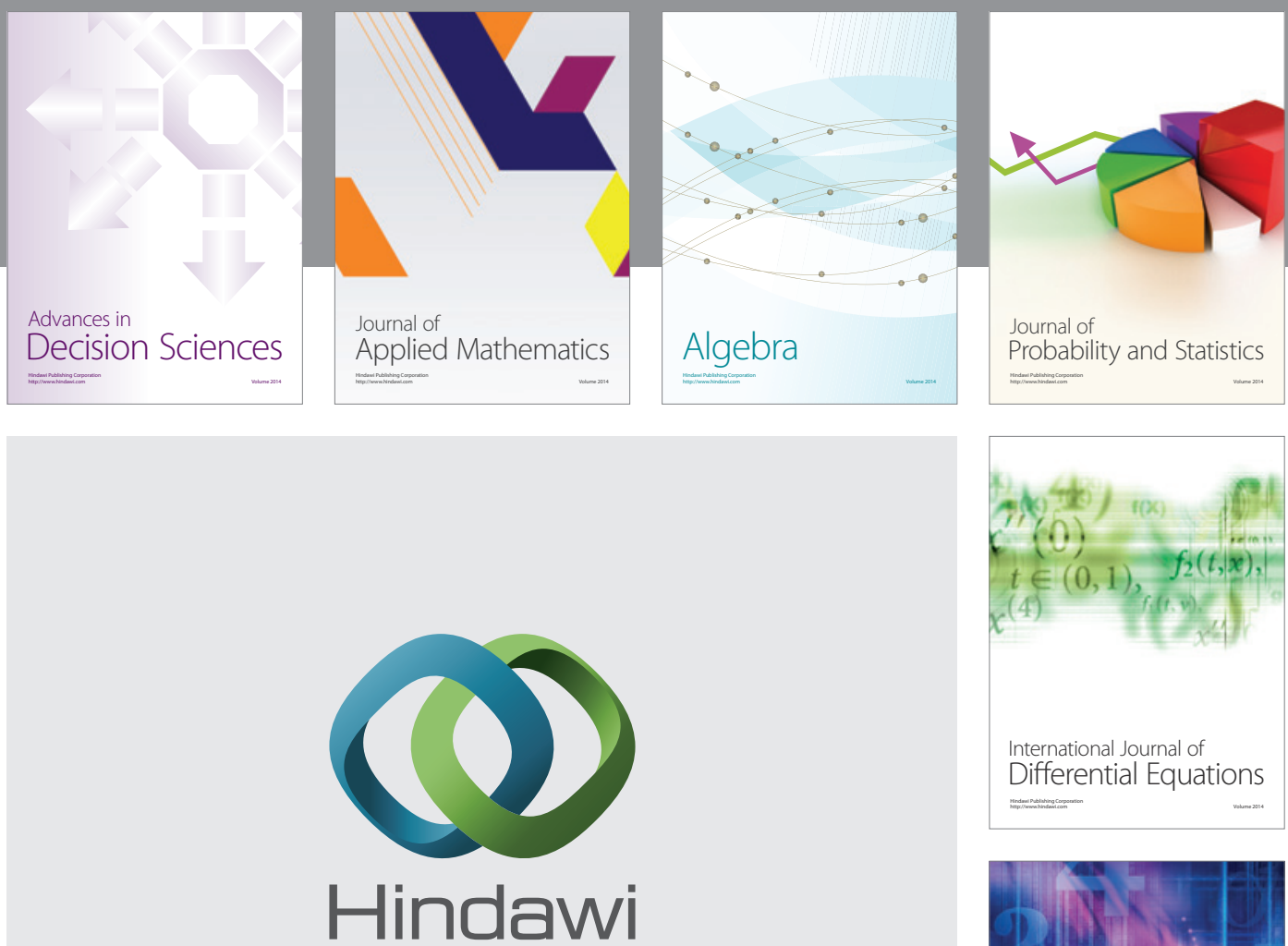

Submit your manuscripts at http://www.hindawi.com
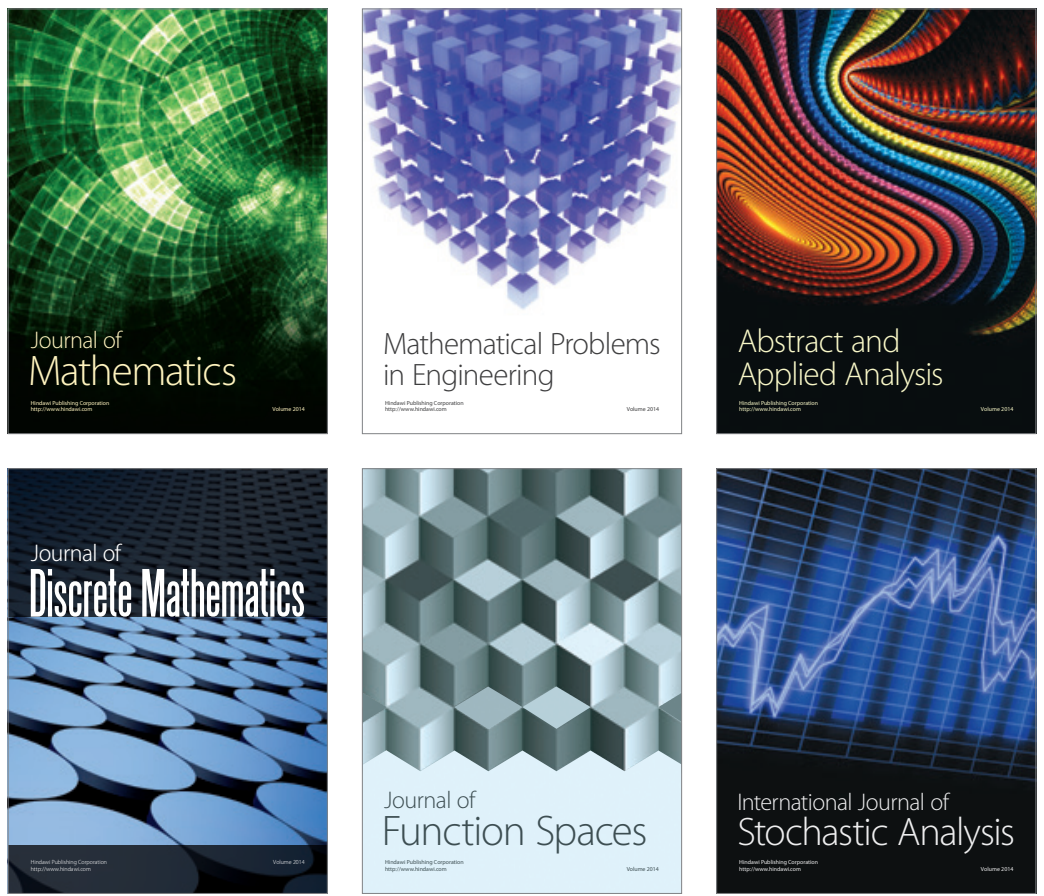

Journal of

Function Spaces

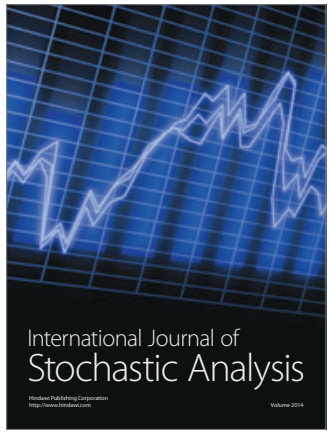

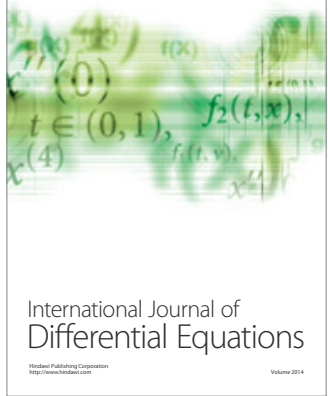
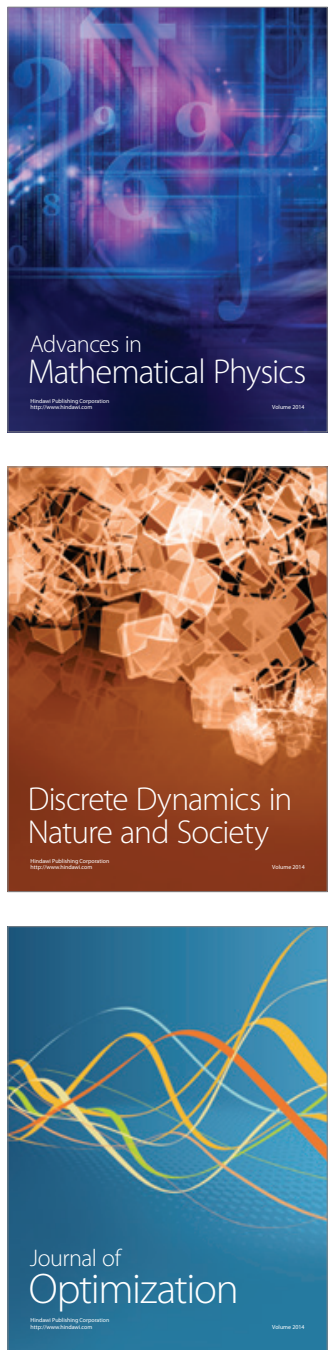